\title{
NOTES
}

\section{Stress Relaxation of Semidilute Polystyrene Solutions. A New Observation with $\Theta$-Solvent and with Blends Containing Very Short Chains}

\author{
Kunihiro OSAKI, ${ }^{*}$ Eiichi TAKatori, Seiichi ShIBASAKI, \\ and Michio KURATA \\ Institute for Chemical Research, Kyoto University, \\ Uji, Kyoto 611, Japan
}

(Received December 14, 1987)

\begin{abstract}
KEY WORDS Stress Relaxation / Polystyrene Solutions / Molecular Weight Distribution / Concentration Fluctuation / $\Theta$-Solvent /
\end{abstract}

In the preceding paper, ${ }^{1}$ we reported the effects of varying short chain components on the relaxation modulus, $G(t)$, of semidilute solutions of polystyrene with bimodal molecular weight distribution. The solution contained two polystyrene samples with molecular weights $M_{\mathrm{S}}$ and $M_{\mathrm{L}}\left(>M_{\mathrm{S}}\right)$ by mass concentrations $c_{\mathrm{S}}$ and $c_{\mathrm{L}}$, respectively. Subscripts $\mathrm{S}$ and $\mathrm{L}$ imply short and long chains, respectively. For a series of solutions with fixed $M_{\mathrm{L}}$ and $c_{\mathrm{L}}\left(8.42 \times 10^{6}\right.$ and $0.038 \mathrm{~g} \mathrm{~cm}^{-3}$, respectively) and varying $M_{\mathrm{S}}\left(9.5 \times 10^{5}-2.89 \times 10^{6}\right)$, the curves obtained by plotting $\log G v s . \log t$ had a common shape over a range of time covering the long time end of the rubbery plateau region and the terminal flow region. In this region, all the curves could be superimposed on that for the monodisperse system with molecular weight $M=M_{\mathrm{L}}$ and concentration $c=c_{\mathrm{L}}$ through parallel shifts along the $\log t$ axis, i.e., by changing the scale of time appropriately. We also observed that when $M_{\mathrm{S}}$ was low, $2.0 \times 10^{5}$, the curve deviated from the common one. The deviation indicated a more gradual decrease in the relaxation spectrum over a wide range of time in contrast with the rather sharp drop at the long end in the monodisperse system.
In the present study, we investigated the effects of very short chains in more detail. We also performed measurements on a solution of ploystyrene of high molecular weight in a $\theta$ solvent since we expected that the deviation might be caused by an enhanced concentration fluctuation of the high $M$ component. The solutions studied are listed in Table I. The molecular weight, $M_{\mathrm{L}}$, and the concentration, $c_{\mathrm{L}}$, of the long chain component were $8.42 \times$ $10^{6}$ and $0.038 \mathrm{~g} \mathrm{~cm}^{-3}$, respectively, throughout in accord with the preceding study. Two samples with $M_{\mathrm{S}}=2.0 \times 10^{5}$ and $5.0 \times 10^{4}$ were used for the short chain component and its concentration, $c_{\mathrm{S}}$, was varied as shown in the table. The good solvent, Aroclor 1248 used also in the preceding study, is a mixture of polychlorinated biphenyls with various degrees of chlorination and was supplied from Monsanto Chemical Company. It is a good solvent for polystyrene. The other solvent, diethylhexyl phthalate (denoted as DOP hereafter), is a $\theta$-solvent for polystyrene. The $\theta$-temperature is $22.0^{\circ} \mathrm{C}$ according to Berry. $^{2}$ The solvent supplied from Nakarai Chemicals, Ltd. (Kyoto) was distilled once before use. Polystyrene samples with $M=$ $5.0 \times 10^{4}$ and $2.0 \times 10^{5}$ are the standard sam- 
Table I. Polystyrene solutions $\mathrm{s}^{\mathrm{a}}$

\begin{tabular}{lccl}
\hline Solvent & $10^{-4} M_{\mathrm{S}}$ & $10^{2} c_{\mathrm{S}} / \mathrm{g} \mathrm{cm}^{-3}$ & $A^{\mathrm{d}}$ \\
\hline \multirow{2}{*}{ Aroclor } & - & $0^{\mathrm{b}, \mathrm{c}}$ & 0 \\
& 5.0 & 3.8 & 0.24 \\
& & 10.0 & 0.24 \\
& 20.0 & $3.8^{\mathrm{c}}$ & 0.18 \\
& & $10.0^{\mathrm{c}}$ & 0.38 \\
& 20.0 & 1.10 \\
\hline \multirow{2}{*}{ DOP } & - & $0^{\mathrm{b}}$ & -0.63 \\
& 5.0 & 10.0 & 0.17 \\
\hline
\end{tabular}

a $M_{\mathrm{L}}=8.42 \times 10^{6}$ and $c_{\mathrm{L}}=0.038 \mathrm{~g} \mathrm{~cm}^{-3}$.

b Solution of a monodisperse polymer with $M=$ $8.42 \times 10^{6}$ and $c=0.038 \mathrm{~g} \mathrm{~cm}^{-3}$.

c Data from ref 1 .

d The shift factor for ordinates of the figures.

ples from Pressure Chemical Company. The quantity $A$ of the last column of Table I appears in the ordinates of the figures.

The linear relaxation modulus, $G(t)$, was measured with a cone-and-plate apparatus. The relaxation modulus was independent of the magnitude of shear when less than 0.7. Measurements for the solutions in Aroclor were performed at several temperatures in the range $20-50^{\circ} \mathrm{C}$ and the data were reduced to a reference temperature, $30^{\circ} \mathrm{C}$, by the method of reduced variables. ${ }^{3}$ Measurements for solutions in DOP were perfomed at $23^{\circ} \mathrm{C}$.

The results are shown in Figures $1-3$; solutions with $M_{\mathrm{S}}=2.0 \times 10^{5}$ in Aroclor in Figure 1, those with $M_{\mathrm{S}}=5.0 \times 10^{4}$ in Aroclor in Figure 2, and those in DOP in Figure 3. The shape of curve in the terminal flow region varied from sample to sample. For convenience of comparison of shape, $\log G$ was plotted against the logarithm of the reduced time, $\log t-A$. The quantity $A$ was determined following the concept that the curve for the bimodal system should overlap the curve for the monodisperse system at the point where it has a slope $-1 / 2$. In this way, data for bimodal systems with $M_{\mathrm{S}}$ larger than $9.5 \times 10^{5}$ lay on a line over the range of long times as reported previously. ${ }^{1}$ The values of $A$ are listed

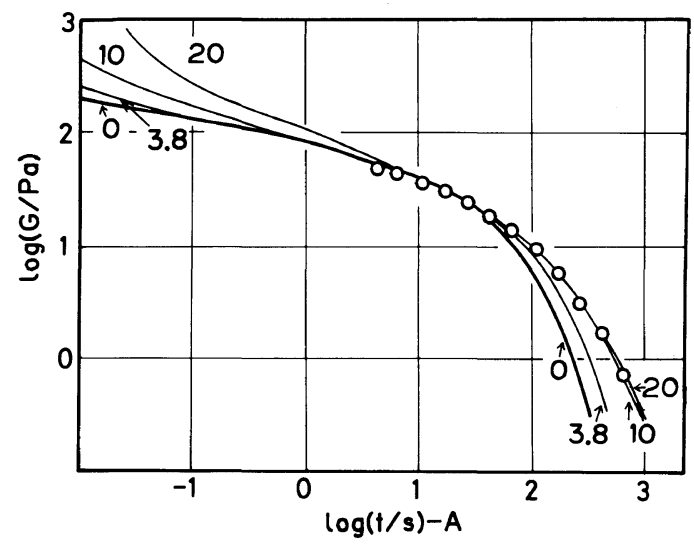

Figure 1. Relaxation modulus for bimodal solutions with $M_{\mathrm{S}}=2.0 \times 10^{5}$ in Aroclor. Thick line represents monodisperse system in Aroclor and circles represent monodisperse system in DOP. Values of $A$ are given in Table I. Numbers in figure indicate $10^{2} c_{\mathrm{S}} / \mathrm{g} \mathrm{cm}^{-3}$.

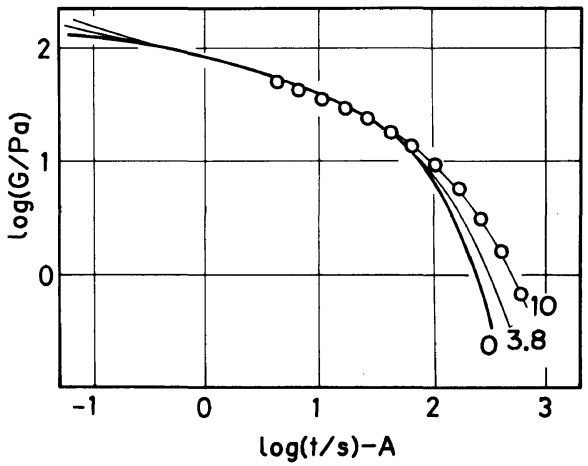

Figure 2. Relaxation modulus for bimodal solutions with $M_{\mathrm{S}}=5.0 \times 10^{4}$ in Aroclor. Thick line represents monodisperse system in Aroclor and circles represent monodisperse system in DOP. Values of $A$ are given in Table I. Numbers in figure indicate $10^{2} c_{\mathrm{s}} / \mathrm{g} \mathrm{cm}^{-3}$.

in Table I. In Figures 1 through 3, we compare the shape of curves at log times, where the reduced time, $\log t-A$, is larger than 1 . Here the short time range is not considered.

In the case of solutions in Aroclor, the slopes of curves in the terminal flow region become less steep with increasing concentration of the short chain component. Thus, the previous observation ${ }^{1}$ is confirmed. The curves may approach a certain limiting curve as the concentration increases; the results for $c_{\mathrm{S}} / \mathrm{g} \mathrm{cm}^{-3}=0.1$ and 0.2 in Figure 1 are very 


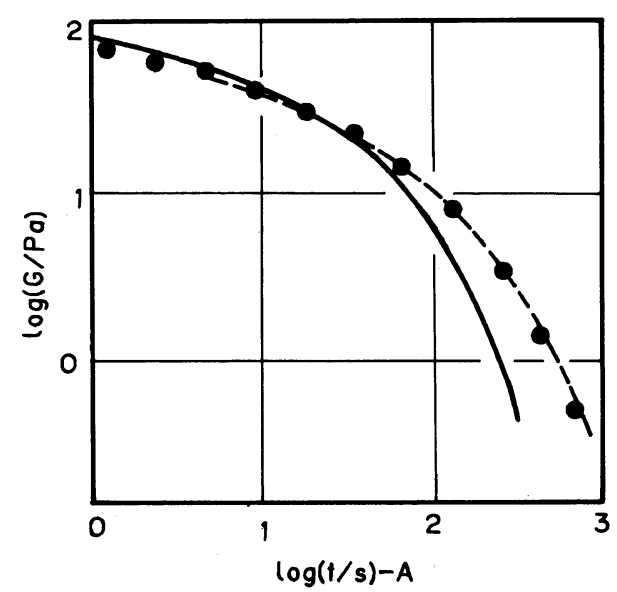

Figure 3. Relaxation modulus for the bimodal solution in DOP (filled circles). Solid and dashed lines represent monodisperse systems in Aroclor and DOP, respectively. Values of $A$ are given in Table $\mathrm{I}$.

close to each other at long times.

The open circles in Figures 1 and 2 represent results for the monodisperse system in DOP plotted in a reduced scale following the above concept. It may be noted that the results for the highest $c_{\mathrm{S}}$ with Aroclor solvent almost agree with those for the monodisperse system with DOP solvent. The agreement in Figure 2 may be fortuitous. On the other hand, the results of Figure 1 may indicate that the high $c_{\mathrm{S}}$ limit in Aroclor solvent corresponds to the monodisperse system in DOP.

The results for the solutions in DOP are shown in Figure 3. The data are plotted following the same concept as in the preceding figures with the monodisperse system in Aroclor as the standard, just for consistency. Obviously the results for the monodisperse and bimodal systems in DOP solvent agree with each other. The short chain component does not affect the shape of curve in the terminal region.

In summary, weakening of the solvent power or the contamination with very short chains makes the relaxation spectrum in the long time range diffuse. For good solvent systems, added short chains make the shape of curve approach that for the monodisperse system in $\theta$-solvent. In $\theta$-solvent, on the other hand, the short chain component does not affect the shape of terminal relaxation curve. The difference in the effects of short chains may imply certain asymptoic behavior in weakening the solvent power or in adding short chains. The diffuse feature of the relaxation spectrum in $\theta$-solvent system or in bimodal systems may be associated with the enhanced inhomogeneity of the segmental mobility or entanglement coupling of long chains. One may conjecture that such imhomogeneity is caused by a possibly larger concentration fluctuation of the long chains compared with that in monodisperse good solvent systems.

It is known that, with increasing concentration, the gross shape of a polymer coil in good solvent approaches that in $\theta$-solvent. On the other hand, the increase in concentration of a monodisperse polymer in good solvent was not accompanied by the diffusing of the relaxation spectrum. Thus, the phenomenon is not related to the gross shape of the polymer chain. We may expect that, in the good solvent, the polymer segments are distributed uniformly, avoiding too close contact. This may be true even at relatively high concentrations where the gross shape of a chain is similar to that in the $\theta$-solvent. It is likely that the diffuse feature of the relaxation spectrum observed here is caused by local concentration fluctuations that may exist in $\theta$-solvent systems or in bimodal polymer mixtures.

Acknowledgement. A polystyrene sample was kindly given us by Toso Corporation.

\section{REFERENCES}

1. K. Osaki, E. Takatori, and M. Kurata, Macromolecules, 20, 1681 (1987).

2. G. C. Berry, J. Chem. Phys., 44, 4500 (1966).

3. J. D. Ferry, "Viscoelastic Properties of Polymers," 3rd ed, Wiley, New York, N. Y., 1980, Chapter 11. 